\title{
Pyrroline-5-Carboxylate Reductase-2 as a Potential Prognostic Biomarker of Colorectal Cancer: A Study Based on Bioinformatics Approaches
}

\section{Haoyi Xiang}

Zhejiang University School of Medicine

\section{Zian Wang}

Zhejiang University School of Medicine

\section{Xuning Shen}

Zhejiang University School of Medicine Sir Run Run Shaw Hospital

\section{Engeng Chen}

Zhejiang University School of Medicine Sir Run Run Shaw Hospital

Wei Chen

Tongde Hospital Of Zhejiang Province

zhangfa song ( $\sim$ songzhangfa@zju.edu.cn)

Zhejiang University https://orcid.org/0000-0001-6301-1813

\section{Research Article}

Keywords: PYCR2, colorectal cancer, invasion, biomarker, prognosis

Posted Date: October 25th, 2021

DOI: https://doi.org/10.21203/rs.3.rs-966708/v1

License: (c) (i) This work is licensed under a Creative Commons Attribution 4.0 International License. Read Full License 


\section{Abstract}

Background: Colorectal cancer (CRC) is associated with high mortality rates. Some studies have attempted to elucidate the molecular mechanisms underlying CRC, and few CRC biomarkers are now used in clinical practice.

Methods: Herein our aim was to identify a new potential prognostic biomarker of CRC, while providing further theoretical basis for studying the pathogenesis of CRC.

Results: PYCR2 expression was to be much higher in CRC tissues than in corresponding non-cancerous tissues, and the prognosis of patients with high PYCR2 expression was significantly poorer than that of those with low PYCR2 expression. Further, PYCR2 showed a high correlation with TRMT61B and CDC23 expression. We found that gene mutations had a significant influence on the prognosis of CRC. PYCR2 siRNA transfection decreased and inhibited the ability of migration and invasion in CRC cells.

Conclusions: To summarize, our findings provide novel insights to facilitate better diagnosis as well as treatment of CRC.

\section{Background}

Colorectal cancer (CRC) is the third most common cancer worldwide and accounts for $9.2 \%$ of new cancer deaths, ranking second among all cancers. The incidence and death rates of CRC have increased in the last decade (including in the Baltic countries, Russia, China, and Brazil).[1] Further, treatment methods, such as surgery, chemotherapy, radiation, and immunotherapy, have rapidly evolved over the past few decades, substantially improving the survival rate of patients with CRC.[2] Approximately 15$25 \%$ patients with CRC also show liver metastasis, and the overall survival of such patients remains poor. [3-5] Early diagnosis and prompt treatment are pivotal to improve the survival rate of patients with CRC. [6] Despite CRC being a critical condition, to date, prognostic ability remains unsatisfactory across the globe.

Pyrroline-5-carboxylate reductase-2 (PYCR2) is one of three human PYCRs that can catalyze the reduction of D1-pyrroline-5-carboxylic acid to proline; further, it is a non-enzymatic antioxidant with the ability to inhibit apoptosis.[7] Under oxidative stress, apoptosis levels have been reported to increase in the absence of PYCR2; moreover, in malignant melanoma, PYCR2 silencing was found to decrease A375 cell proliferation and induce autophagy via the AMPK/mTOR pathway.[8] In addition, Gao et al. identified PYCR2 to be a prognostic biomarker of hepatocellular carcinoma; they found that PYCR2 was related to proteomic subgrouping and involved in the metabolic reprogramming of hepatocellular carcinoma.[9]

Based on the aforementioned data, we hypothesized that PYCR2 is closely related to CRC and that it plays a crucial role in CRC development. Herein we analyzed several databases to investigate the expression levels of PYCR2 and related genes in CRC. Our aim was to elucidate the role of PYCR2 in CRC development and to determine whether PYCR2 can be used as a prognostic biomarker of CRC. 


\section{Methods}

\section{Exploring PYCR2 prognostic ability and expression pattern in $\mathrm{CRC}$}

ENCORI Pan-Cancer Analysis Platform[10] (http://starbase.sysu.edu.cn/panCancer.php) is designed for decoding pan-cancer networks of IncRNAs, miRNAs, pseudogenes, snoRNAs, RNA-binding proteins, and protein-coding genes by assessing their expression profiles across 32 cancer types integrated from The Cancer Genome Atlas (TCGA) project. In this study, we used this platform to study PYCR2 expression pattern and prognostic ability in CRC.

\section{Target gene prediction and data screening}

To improve the efficiency of target gene prediction, we used four databases: STRING[11, 12] (https://string-db.org/), BioGRID (https://thebiogrid.org/), IntAct[13] (https://www.ebi.ac.uk/intact/), and GeneMANIA[14] (https://genemania.org/). Further, Venn diagram was created through a web tool (http://bioinformatics.psb.ugent.be/webtools/Venn/) and target genes chosen which were overlapped in at least 2 out of 4 databases to enhance the accuracy of the prediction.

\section{Functional analysis of PYCR2 overlapping target genes}

To further elucidate the biological function of PYCR2 overlapping target genes in CRC, we performed gene ontology (GO) enrichment analysis using Metascape[15] (http://metascape.org/gp/index.html).

\section{Determining the prognostic significance of PYCR2 overlapping target genes}

ENCORI and GEPIA[16] (http://gepia.cancer-pku.cn/) were applied to determine whether the expression levels of PYCR2 overlapping target genes varied between CRC and non-cancerous tissues and whether they affected the prognosis of CRC.

Assessing the co-expression of PYCR2 and related genes and determining the mutational status of target genes

cBioPortal[17, 18] (http://www.cbioportal.org/), a web-based tool to explore, visualize, and analyze multidimensional cancer genomics data, was used to analyze the co-expression of PYCR2 and related genes. $P<0.05$ was the cut-off criterion. Further, the mutational status of the target genes of PYCR2 in $\mathrm{CRC}$ was investigated using cBioPortal.

\section{Comparing prognosis between patients with and without gene mutations}

The International Cancer Genome Consortium (ICGC)[19] (https://dcc.icgc.org/) serves to catalog largescale cancer genome studies in tumors from 50 distinct cancer types/subtypes, containing clinical data 
as well as data pertaining to abnormal gene expression, somatic mutations, and epigenetic modifications. We used this portal to compare prognosis between patients with and without gene mutations.

\section{Cell culture}

HCT116, HT29, and LoVo CRC cell lines were obtained from the American Type Culture Collection (Manassas, VA, USA). LoVo and HT29 cells were maintained in 1640 (HyClone), 10\% FBS (Gibco), and 100 units/mL penicillin, HCT116 cells were maintained in McCoy's 5A (HyClone), 10\% FBS (Gibco), and 100 units/mL penicillin.

\section{Cell transfection}

HT29, LoVo, and HCT116 cells were seeded into a 6 well plate and cultivated at $37^{\circ} \mathrm{C}$ and $5 \% \mathrm{CO}_{2}$. Their growth was closely monitored until they reached a fusion rate of $80 \%$, and they were then transfected with siRNA (final concentration $=100 \mathrm{nM}$; Santa Cruz, Texas, USA) in the presence of liposome 2000 (Invitrogen, California, USA). Briefly, Liposome diluted to $5 \mu \mathrm{l} /$ well, and then siRNA was diluted at a calculated concentration. We incubated them for 5 minutes separately, and incubated the mixture proportioned for 15 minutes. The mixture was added to the cells and the 6-well plate placed into the incubator. After $8 \mathrm{~h}$, the growth medium was changed to fresh media, and the cells were further incubated at $37^{\circ} \mathrm{C}$ for $24 \mathrm{~h}\left(5 \% \mathrm{CO}_{2}\right)$. The cells were subsequently collected for further analyses.

\section{Reverse transcription-quantitative PCR}

TRIzol ${ }^{\circledR}$ was used to extract total RNA from HCT116, HT29, and LoVo cells, as per manufacturer instructions. Reverse transcription was performed using the PrimeScript RT reagent kit (Takara Bio, Inc.). rRNA amplification was performed on a C1000 Thermal Cycler Detection System (Bio-Rad Laboratories, Inc.) in triplicate, with the reaction mixture (total volume $=50 \mu \mathrm{L}$ ) comprising $1 \mathrm{ng} c D N A, 125 \mathrm{nM}$ forward and reverse primers, and $25 \mu \mathrm{L} 2 \mathrm{X} \mathrm{SYBR}^{\circledR}$ Premix Ex Taq ${ }^{\mathrm{TM}}$ (Takara Bio, Inc.). A NanoDrop 2000 Spectrophotometer (Thermo) was used for RNA quantification, with the A260/280 ratio indicating RNA purity. GAPDH was used as the internal control. The cycling conditions were as follows: initial denaturation at $95^{\circ} \mathrm{C}$ for $1 \mathrm{~min}$, followed by 42 cycles of $95^{\circ} \mathrm{C}$ for $15 \mathrm{sec}, 56^{\circ} \mathrm{C}$ for $25 \mathrm{sec}$, and $72^{\circ} \mathrm{C}$ for 30 sec. The following primers were used: GAPDH-F: 5'-AGCCACATCGCTCAGAACAC-3', GAPDH-R: 5'GAGGCATTGCTGATGATCTTG-3'; PYCR2-F: 5'- CTGTCCCAGGTGTCTAAGGG-3', PYCR2-R: 5'CTTGCTCCTCAGCACAGAAC-3'. The relative mRNA expression levels were quantified using the $2^{-\triangle \triangle C q}$ method.

\section{Wound healing}

We inoculated approximately $3 \times 10^{5}$ cells/well into a 6 -well plate, with the exact number varying on a cell-to-cell basis. The cells were intervened with siRNA separately. The next day, the plates were scratched vertically using a pipette tip. Scratches were ensured to be straight using a ruler. The cells were washed with PBS. The scratched cells were then removed and a serum-free medium was added into the well. The 
plates were subsequently incubated at $37^{\circ} \mathrm{C}$ and $5 \% \mathrm{CO}_{2}$. Samples were collected at 0,24 , and $48 \mathrm{~h}$, and images were then captured.

\section{Transwell assay}

Matrigel $(8-12 \mathrm{mg} / \mathrm{ml})$ was placed, which was stored at $-20{ }^{\circ} \mathrm{C}$, onto the ice to melt (keeping the temperature at $\left.2{ }^{\circ} \mathrm{C} \otimes 8^{\circ} \mathrm{C}\right)$. Matrigel $(100 \mu \mathrm{l})$ was aspirated with a pipette tip precooled on ice and expelled into precooled $3000 \mu \mathrm{l}$ serum-free medium (diluted at 1:30-1:40, total volume diluted according to the number of wells), and mixed thoroughly. The diluted $100 \mu$ l Matrigel above was added into the upper chamber of the Transwell plate to cover the whole polycarbonyl ester membrane. The Matrigel was polymerized into gelatin at $37^{\circ} \mathrm{C}$ for 60 minutes. Any remaining liquid medium on the top was discarded carefully.

The pretreated groups of cells were prepared into single-cell suspension $\left(5 \times 10^{\wedge} 5 \mathrm{cell} / \mathrm{s} / \mathrm{ml}\right)$ with serumfree medium in the regular way. This suspension $(200 \mu \mathrm{l})$ was added to the upper chamber of the Transwell culture plate, and $700 \mu \mathrm{l} 10 \%$ FBS was added to the lower chamber. The plate was then incubated at $37^{\circ} \mathrm{C}$ and $5 \% \mathrm{CO}_{2}$ for $48 \mathrm{~h}$. Subsequently, the cells were fixed in $4 \%$ paraformaldehyde for 30 min and then stained with $0.1 \%$ crystal violet for 3 min.

To measure the number of cells that were able to penetrate the film in the upper chamber, the unpenetrated cells on the upper surface of the film and the Matrigel in the chamber were gently wiped off with a wet cotton swab. Finally, images were captured, and the cells were counted.

\section{Statistical analysis}

GraphPad Prism v9.00 was used for statistical analyses, and differences were determined with Student's $t$-tests. $\mathrm{P}<0.05$ indicated statistical significance.

\section{Results}

\section{PYCR2 expression pattern in CRC}

As evident from Fig. 1, starBase v3.0 project(https://starbase.sysu.edu.cn/index.php) showed that the expression level of PYCR2 in CRC tissues was distinctly higher than that in non-cancerous tissues ( $p=$ 6.0e-13), and the prognosis of patients with CRC with high expression levels of PYCR2 was worse than that of those with low expression levels of PYCR2 $(p<0.05)$. These results indicated that PYCR2 plays an important role in CRC and may be an oncogene that promotes CRC progression.

\section{Target gene prediction and data screening}

The analysis involving STRING, BioGRID, IntAct, and GeneMANIA led to the identification of 10, 64, 44, and 19 genes, respectively. In total, 137 PYCR2-related genes were identified (Table 1). To increase the 
accuracy of target gene prediction, we selected genes that overlapped in at least two of the four databases (Fig. 2), and ultimately, 36 potentially related genes were identified (Table 2).

Table 1

CRC-related genes identified using STRING, BioGRID, IntAct, and GeneMANIA

\begin{tabular}{|c|c|}
\hline Databases & Gene names \\
\hline IntAct & $\begin{array}{l}\text { LRRK2 COX4I1 MCC MYO1F CUL3 SFXN1 HSPD1 SIRT4 HSCB AIFM1 BCL2L14 CIC } \\
\text { CEP162 AHNAK AURKAIP1 DLST RELA TNFRSF10A SKIL SCO1 PDK1 SYDE1 IRF2 } \\
\text { NT5C3A RAC1 MGST3 TRMT61B DLD VCAM1 SHC1 SKA1 CDC16 MRPL58 COX14 } \\
\text { AHNAK2 SH2D3C PYCR1 PYCR3 FASTKD3 CDC23 NUDT5 PDHA1 FKBPL L1TD1 }\end{array}$ \\
\hline STRING & PYCR1 NUDT5 P4HA1 P4HA3 P4HA2 LAP3 PYCRL PIG6 ALDH4A1 ALDH18A1 \\
\hline BioGRID & $\begin{array}{l}\text { PYCR1 MY01F CEP162 AHNAK2 SKA1 NUDT5 SKA3 MYO18A PYCRL PDK1 DUSP14 } \\
\text { COX14 SFXN1 AURKAIP1 HSPD1 SCO1 MRM1 AIFM1 MYC COX4I1 TRMT61B } \\
\text { RECQL4 TERF2 CLPP COL11A1 HSPA4 CHD3 ME2 PAICS PRDX4 IRF2 BCL2L14 } \\
\text { NT5C3A TNFRSF10A BIRC3 TRIM28 ESR2 TNS3 TNIP2 CDC16 EBNA-LP CUL3 } \\
\text { KCND3 CIC APP MDC1 VCAM1 SKIL EGFR NTRK1 CDC23 AR TRIM14 CDC14 CDC16 } \\
\text { ANAPC2 CEP85L NF2 NPLOC4 ICT1 AHNAK DLD DLST PDHA1 PLEKHA4 }\end{array}$ \\
\hline GeneMANIA & $\begin{array}{l}\text { LRRK2 PYCRL KCND3 MY01F AR SKA3 NOXRED1 CEP85L MDM2 PPAN NUDT } 5 \\
\text { MCM3AP STEAP2 RECQL5 TERF2 MPV17L2 STEAP4 SKA1 STEAP3 }\end{array}$ \\
\hline
\end{tabular}


Table 2

Target genes overlapping in at least two of the four databases

\begin{tabular}{|c|c|c|}
\hline Databases & $\begin{array}{l}\text { Number } \\
\text { of } \\
\text { genes }\end{array}$ & Gene names \\
\hline $\begin{array}{l}\text { BioGRID } \\
\text { GeneMANIA } \\
\text { IntAct } \\
\text { STRING }\end{array}$ & 1 & NUDT5 \\
\hline $\begin{array}{l}\text { BioGRID } \\
\text { IntAct } \\
\text { STRING }\end{array}$ & 1 & PYCR1 \\
\hline $\begin{array}{l}\text { BioGRID } \\
\text { GeneMANIA } \\
\text { STRING }\end{array}$ & 1 & PYCRL \\
\hline $\begin{array}{l}\text { BioGRID } \\
\text { GeneMANIA } \\
\text { IntAct }\end{array}$ & 2 & SKA1 MY01F \\
\hline $\begin{array}{l}\text { BioGRID } \\
\text { IntAct }\end{array}$ & 25 & $\begin{array}{l}\text { CDC16 HSPD1 AURKAIP1 SFXN1 PDHA1 CUL3 AIFM1 TNFRSF10A } \\
\text { VCAM1 SCO1 AHNAK2 PDK1 DLST CDC23 AHNAK TRMT61B IRF2 } \\
\text { NT5C3A DLD CIC CEP162 BCL2L14 COX14 COX4I1 SKIL }\end{array}$ \\
\hline $\begin{array}{l}\text { BioGRID } \\
\text { GeneMANIA }\end{array}$ & 5 & SKA3 TERF2 CEP85L KCND3 AR \\
\hline $\begin{array}{l}\text { GeneMANIA } \\
\text { IntAct }\end{array}$ & 1 & LRRK2 \\
\hline
\end{tabular}

\section{Functional analysis of the overlapping target genes}

Through GO enrichment analysis, we studied the biological roles of the 36 aforementioned genes in CRC (Table 3). The target genes of PYCR2 were mainly enriched in biological processes such as positive

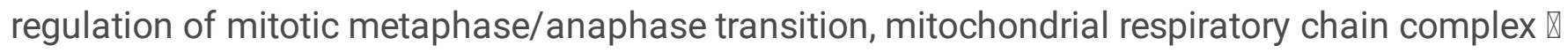
assembly, apoptotic signaling pathway, response to hydrogen peroxide, regulation of oxidoreductase activity, and mitochondrial gene expression. The apoptotic signaling pathway was one of the most enriched pathways. 
Table 3

Gene ontology enrichment analysis of overlapping target genes

\begin{tabular}{|c|c|c|c|c|c|c|}
\hline GO & Category & Description & Count & $\%$ & $\log 10(P)$ & $\log 10(q)$ \\
\hline $\begin{array}{l}\text { R-HAS- } \\
1428517\end{array}$ & $\begin{array}{l}\text { Reactome } \\
\text { Gene Sets }\end{array}$ & $\begin{array}{l}\text { The citric acid(TCA)cycle } \\
\text { and respiratory electron } \\
\text { transport }\end{array}$ & 7 & 18.92 & -8.11 & -3.79 \\
\hline GO:0045842 & $\begin{array}{l}\text { GO } \\
\text { Biological } \\
\text { Processes }\end{array}$ & $\begin{array}{l}\text { Positive regulation of } \\
\text { mitotic metaphase } \\
\text { /anaphase transition }\end{array}$ & 3 & 8.11 & -6.04 & -2.39 \\
\hline G0:0033617 & $\begin{array}{l}\text { GO } \\
\text { Biological } \\
\text { Processes }\end{array}$ & $\begin{array}{l}\text { Mitochondrial respiratory } \\
\text { chain complex } \square \\
\text { assembly }\end{array}$ & 3 & 8.11 & -5.38 & -2.07 \\
\hline GO:0097190 & $\begin{array}{l}\text { GO } \\
\text { Biological } \\
\text { Processes }\end{array}$ & $\begin{array}{l}\text { Apoptotic signaling } \\
\text { pathway }\end{array}$ & 7 & 18.92 & -4.51 & -1.70 \\
\hline GO:0042542 & $\begin{array}{l}\text { GO } \\
\text { Biological } \\
\text { Processes }\end{array}$ & $\begin{array}{l}\text { Response to hydrogen } \\
\text { peroxide }\end{array}$ & 4 & 10.81 & -4.16 & -1.44 \\
\hline GO:0051341 & $\begin{array}{l}\text { GO } \\
\text { Biological } \\
\text { Processes }\end{array}$ & $\begin{array}{l}\text { Regulation of } \\
\text { oxidoreductase activity }\end{array}$ & 3 & 8.11 & -3.12 & -0.74 \\
\hline GO:0140053 & $\begin{array}{l}\text { GO } \\
\text { Biological } \\
\text { Processes }\end{array}$ & $\begin{array}{l}\text { Mitochondrial gene } \\
\text { expression }\end{array}$ & 3 & 8.11 & -2.7 & -0.41 \\
\hline
\end{tabular}

Prognostic significance of the overlapping target genes

Of 36, the expression levels of four genes-AHNAK2, CDC23, TRMT61B, and DLD-were significantly altered in CRC tissues as compared with those in non-cancerous tissues $(p<0.05)$ (Fig. 3A). Further, the prognosis of these four genes was also significantly altered in $\mathrm{CRC}$ and non-cancerous tissues through ENCORI and Gepia ( $<$ 0.05) (Fig. 3B). These data suggested that AHNAK2, CDC23, TRMT61B, and DLD have a certain effect on CRC and that PYCR2 may have an effect on CRC via them, though the specific mechanism remains unclear.

\section{Co-expression of PYCR2 and related genes}

Our co-expression analysis (Table 4) revealed the existence of a close association between PYCR2 and TRMT61B as well as CDC23 in CRC. 
Table 4

Co-expression between PYCR2 and

related genes

\section{Gene name Gene name p value \\ TRMT61B PYCR20.008 \\ CDC23 PYCR2 0.045 \\ Mutation of PYCR2 target genes in CRC}

Due to the high frequency of some genes amplification in $\mathrm{CRC}$, the role played by gene mutation may be greatly changed. We used cBioPortal to explore gene-specific changes in TCGA CRC data (Fig. 4). The genes such as MYO1F, AHNAK2, TNFRSF10A, and CIC showed a high mutation frequency in CRC.

\section{Comparison of prognosis between patients with and without gene mutations}

Using the ICGC database, we determined the prognosis of patients with and without target gene mutations in $\mathrm{CRC}$ and found that $\mathrm{CIC}$ and MYO1F had a significant impact on the prognosis $(\mathrm{p}<0.05)$. Moreover, the overall survival curves of CDC16, DLD, IRF2, and FASTKD showed a significant change. However, $p$ value was not statistically significant, which could be due to the insufficient samples size (Fig. 5). We believe that the mutation of these target genes is of critical significance for CRC patients.

\section{PYCR2 promotes both proliferation and invasion in CRC}

To explore the biological role of PYCR2 in CRC, we knocked down PYCR2 expression in HCT116, LoVo, and HT29 cells in vitro $(\mathrm{p}<0.05)($ Fig. $6 \mathrm{~A}, \mathrm{~B})$. Wound healing assay results indicated that the migration of HCT116, LoVo, and HT29 cells decreased when PYCR2 was knocked down (Fig. 6C). Further, transwell assay results validated that in comparison with the negative control group, cell invasion ability was inhibited after PYCR2 expression was knocked down in HCT116, LoVo, and HT29 cells (Fig. 6D). Statistical analyses revealed that the number of migratory cells showed an obvious decrease after knocking down PYCR2 in HCT116, LoVo, and HT29 cells (Fig. 6E). Collectively, our data indicated that PYCR2 has a momentous role in the development process of CRC.

\section{Discussion}

CRC majorly contributes to cancer-related deaths globally, and its incidence rates are approximately 3fold higher in transitioned versus transitioning countries. However, low human development index countries have a higher mean case fatality rate while the difference in mortality rate is smaller. ${ }^{[1]}$ Considering that traditional methods to treat CRC, including chemotherapy and radiotherapy, remain unsatisfactory, new methods, such as tumor-specific targeted therapy, have emerged. Identifying novel targets has become an indisputable part of tumor-specific targeted therapy.[20] These insights indicate 
that it is vital to find an independent predictive factor to evaluate the prognosis of CRC. PYCR2 is closely related to oxidative stress and cell apoptosis; $[8,21]$ it also plays a key role in tumor energy metabolism, and thus, PYCR2 appears to represent a new group of potential target molecules for tumor treatment. To comprehensively understand the relationship between PYCR2 and CRC prognosis, herein we performed bioinformatics analyses based on TCGA database, and verified our results in vitro using a wound healing assay and Transwell test.

To the best of our knowledge, we for the first time report the abnormal expression of PYCR2 in CRC, which PYCR2 expression levels were notably higher in CRC tissues than in corresponding non-cancerous tissues, based on TCGA dataset validation. We herein predicted PYCR2 target genes $(n=36)$; overlapping target genes were detected by $\mathrm{GO}$ enrichment analysis, which revealed that the apoptotic signaling pathway was significantly enriched. Apoptotic signaling is essential to maintain a healthy balance between cell death and survival and to maintain genomic integrity. The imbalance between pro- and antiapoptotic proteins promotes tumorigenesis by reducing the apoptosis of malignant tumor cells.[22] The function of these target genes of PYCR2 in the biological process was investigated, and we found that the expression status of AHNAK2, CDC23, TRMT61B, and DLD in CRC was remarkably different from that in corresponding non-cancerous tissues; furthermore, a notable difference was present in overall survival rate between the high and low expression groups. AHNAK2 has been reported to promote migration, invasion, and epithelial-mesenchymal transformation of lung adenocarcinoma cells via the transforming growth factor- $\beta / S m a d 3$ pathway.[23] Moreover, TRMT61B and CDC23 were co-expressed in CRC $(p<0.05)$. TRMT61B is a type of methyltransferase. RNA methyltransferases are closely associated with oncogene transcription and expression. TRMT61B also participates in cancer cell proliferation and tumor initiation, progression, and metastasis.[24] CDC23 is a key regulator of mitotic process. [25] We accordingly analyzed the status of these genes in CRC. The mutation frequency of some genes, such as MY01F, AHNAK2, TNFRSF10A, LRRK2, and CIC, was found to be high. We also analyzed gene mutations and prognosis in patients with $\mathrm{CRC}$, and the results indicated that the prognosis of patients with MYO1F and $\mathrm{CIC}$ gene mutations was significantly different from that of those without mutations. In addition, the prognosis of patients with CDC16, DLD, IRF2, and FASTKD gene mutations was significantly different from that of those without mutations, which shows high importance of these genes in tumorigenesis. TNFRSF10A reportedly mediates endoplasmic reticulum stress-induced apoptosis in human lung cancer cells in a DDIT3 activation-dependent manner.[26] As for MY01F, mutant MY01F has been found to alter the mitochondrial network and induce tumor proliferation in thyroid cancer.[27]

To further determine the impact of PYCR2 on CRC prognosis, we knocked down PYCR2 expression. Wound healing and Transwell assay results showed that in comparison with the negative control group, cell invasion ability was significantly inhibited after PYCR2 expression was knocked down in HCT116, LoVo, and HT29 cells. We thus report that in vitro, PYCR2 plays a salient role participating in the invasion of CRC cells.

\section{Conclusions}


In this study, we used several online bioinformatics platforms and online tools to systematically analyze the expression of PYCR2 and related genes in CRC; further, we assessed their prognostic significance. For the first time, we report that PYCR2 is a potential prognostic biomarker of CRC. Our findings also shed light on the importance of the expression of PYCR2 and related genes, as well as the potential role of PYCR2-related pathways in the progression of various human cancers. Our analyses provide valuable insights into PYCR2 as a novel biomarker, presenting it as a potential therapeutic target for several human cancers and contributing to the translation of genomic knowledge into clinical practice. Further studies are warranted to elucidate the specific role, detailed molecular mechanisms, and clinical significance of PYCR2 in tumor progression and prognosis.

\section{Declarations}

\section{Ethics approval and consent to participate}

No applications.

\section{Consent for publication}

Not applicable (Date from starBase v3.0)

\section{Authors' contributions}

HX, WC and ZS conceived the idea; HX performed the experiments; ZW, XS and EC analyzed the data; ZS wrote the manuscript. All authors have read and approved the final version of the manuscript.

\section{Acknowledgements}

We are grateful to the contributors of data to ENCORI, TCGA, STRING, BioGRID, IntAct, GeneMANIA, Metascape, GEPIA, cBioPortal, and ICGC.

\section{Funding}

This study was supported by the National Natural Science Foundation of China (No.81771502, 81701820), the Natural Science Foundation of Zhejiang Province (No. LH19H160001) and the Department of Health of Zhejiang Province (No. 2018KY473 and 2018PY0250).

\section{Competing interests}

The authors declare that they have no competing $f$ interests.

\section{Availability of data and materials}

We declare that all data supporting the conclusions of the study. 


\section{References}

1. Bray F, Ferlay J, Soerjomataram I, Siegel RL, Torre LA, Jemal A. Global cancer statistics 2018: GLOBOCAN estimates of incidence and mortality worldwide for 36 cancers in 185 countries. CA Cancer J Clin. 2018;68:394-424.

2. Dekker E, Tanis P, Vleugels J, Kasi P, Wallace M. Colorectal cancer. Lancet. 2019;394:1467-80.

3. Rahbari N, Kedrin D, Incio J, Liu H, Ho W, Nia H, Edrich C, Jung K, Daubriac J, Chen I, et al. Anti-VEGF therapy induces ECM remodeling and mechanical barriers to therapy in colorectal cancer liver metastases. Science translational medicine. 2016;8:360ra135.

4. Lin J, Piper M, Perdue L, Rutter C, Webber E, O'Connor E, Smith N, Whitlock E. Screening for Colorectal Cancer: Updated Evidence Report and Systematic Review for the US Preventive Services Task Force. JAMA. 2016;315:2576-94.

5. Tejpar S, Shen L, Wang X, Schilsky R. Integrating biomarkers in colorectal cancer trials in the West and China. Nature reviews Clinical oncology. 2015;12:553-60.

6. Siegel R, Miller K, Goding Sauer A, Fedewa S, Butterly L, Anderson J, Cercek A, Smith R, Jemal A. Colorectal cancer statistics, 2020. CA: a cancer journal for clinicians 2020, 70:145-164.

7. Nakayama T, Al-Maawali A, El-Quessny M, Rajab A, Khalil S, Stoler J, Tan W, Nasir R, Schmitz-Abe K, Hill R, et al. Mutations in PYCR2, Encoding Pyrroline-5-Carboxylate Reductase 2, Cause Microcephaly and Hypomyelination. Am J Hum Genet. 2015;96:709-19.

8. Ou R, Zhang X, Cai J, Shao X, Lv M, Qiu W, Xuan X, Liu J, Li Z, Xu Y. Downregulation of pyrroline-5carboxylate reductase- 2 induces the autophagy of melanoma cells via AMPK/mTOR pathway. Tumor Biology. 2015;37:6485-91.

9. Gao Q, Zhu H, Dong L, Shi W, Chen R, Song Z, Huang C, Li J, Dong X, Zhou Y, et al. Integrated Proteogenomic Characterization of HBV-Related Hepatocellular Carcinoma. Cell. 2019;179:561-77 e522.

10. Li J, Liu S, Zhou H, Qu L, Yang J. starBase v2.0: decoding miRNA-ceRNA, miRNA-ncRNA and proteinRNA interaction networks from large-scale CLIP-Seq data. Nucleic acids research. 2014;42:D92-7.

11. Szklarczyk D, Gable A, Lyon D, Junge A, Wyder S, Huerta-Cepas J, Simonovic M, Doncheva N, Morris $J$, Bork P, et al. STRING v11: protein-protein association networks with increased coverage, supporting functional discovery in genome-wide experimental datasets. Nucleic acids research. 2019;47:D607-13.

12. Szklarczyk D, Morris J, Cook H, Kuhn M, Wyder S, Simonovic M, Santos A, Doncheva N, Roth A, Bork $P$, et al. The STRING database in 2017: quality-controlled protein-protein association networks, made broadly accessible. Nucleic acids research. 2017;45:D362-8.

13. Orchard S, Ammari M, Aranda B, Breuza L, Briganti L, Broackes-Carter F, Campbell N, Chavali G, Chen $\mathrm{C}$, del-Toro $\mathrm{N}$, et al. The MlntAct project-IntAct as a common curation platform for 11 molecular interaction databases. Nucleic acids research. 2014;42:D358-63. 
14. Warde-Farley D, Donaldson S, Comes O, Zuberi K, Badrawi R, Chao P, Franz M, Grouios C, Kazi F, Lopes $\mathrm{C}$, et al. The GeneMANIA prediction server: biological network integration for gene prioritization and predicting gene function. Nucleic acids research. 2010;38:W214-20.

15. Zhou Y, Zhou B, Pache L, Chang M, Khodabakhshi A, Tanaseichuk O, Benner C, Chanda S. Metascape provides a biologist-oriented resource for the analysis of systems-level datasets. Nature communications. 2019;10:1523.

16. Tang Z, Li C, Kang B, Gao G, Li C, Zhang Z. GEPIA: a web server for cancer and normal gene expression profiling and interactive analyses. Nucleic acids research. 2017;45:W98-102.

17. Gao J, Aksoy B, Dogrusoz U, Dresdner G, Gross B, Sumer S, Sun Y, Jacobsen A, Sinha R, Larsson E, et al. Integrative analysis of complex cancer genomics and clinical profiles using the cBioPortal. Sci Signal. 2013;6:pl1.

18. Cerami E, Gao J, Dogrusoz U, Gross B, Sumer S, Aksoy B, Jacobsen A, Byrne C, Heuer M, Larsson E, et al. The cBio cancer genomics portal: an open platform for exploring multidimensional cancer genomics data. Cancer discovery. 2012;2:401-4.

19. Zhang J, Bajari R, Andric D, Gerthoffert F, Lepsa A, Nahal-Bose H, Stein L, Ferretti V. The International Cancer Genome Consortium Data Portal. Nature biotechnology. 2019;37:367-9.

20. Piawah S, Venook A. Targeted therapy for colorectal cancer metastases: A review of current methods of molecularly targeted therapy and the use of tumor biomarkers in the treatment of metastatic colorectal cancer. Cancer. 2019;125:4139-47.

21. Chen C, Dickman M. Proline suppresses apoptosis in the fungal pathogen Colletotrichum trifolii. Proc Natl Acad Sci USA. 2005;102:3459-64.

22. Goldar S, Khaniani M, Derakhshan S, Baradaran B. Molecular mechanisms of apoptosis and roles in cancer development and treatment. Asian Pacific journal of cancer prevention: APJCP. 2015;16:2129-44.

23. Liu G, Guo Z, Zhang Q, Liu Z, Zhu D. AHNAK2 Promotes Migration, Invasion, and EpithelialMesenchymal Transition in Lung Adenocarcinoma Cells via the TGF- $\beta$ /Smad3 Pathway. OncoTargets therapy. 2020;13:12893-903.

24. Lan Q, Liu P, Haase J, Bell J, Hüttelmaier S, Liu T. The Critical Role of RNA mA Methylation in Cancer. Cancer research. 2019;79:1285-92.

25. Achari C, Winslow S, Ceder Y, Larsson C. Expression of miR-34c induces G2/M cell cycle arrest in breast cancer cells. BMC Cancer. 2014;14:538.

26. Li T, Su L, Lei Y, Liu X, Zhang Y, Liu X. DDIT3 and KAT2A Proteins Regulate TNFRSF10A and TNFRSF10B Expression in Endoplasmic Reticulum Stress-mediated Apoptosis in Human Lung Cancer Cells. J Biol Chem. 2015;290:11108-18.

27. Diquigiovanni C, Bergamini C, Evangelisti C, Isidori F, Vettori A, Tiso N, Argenton F, Costanzini A, Iommarini L, Anbunathan $\mathrm{H}$, et al. Mutant MY01F alters the mitochondrial network and induces tumor proliferation in thyroid cancer. International journal of cancer. 2018;143:1706-19. 


\section{Figures}
A
B
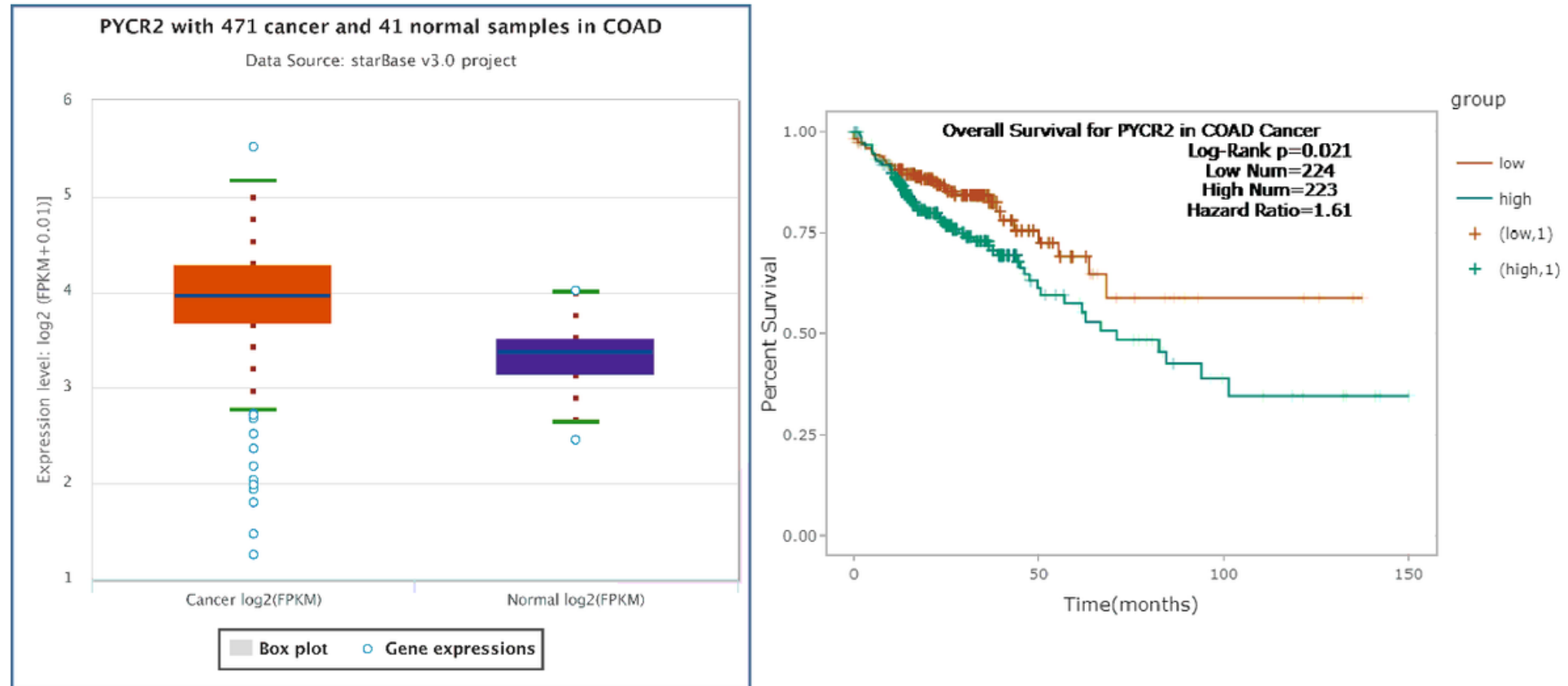

$\mathrm{P}=6.0 \mathrm{e}-13$

$\mathrm{P}=0.021$

\section{Figure 1}

PYCR2 expression and prognosis pattern in CRC. (A) Box with color of orange represents the group of $\mathrm{CRC}$, while the box with purple represents the normal group. PYCR2 expression level in CRC tissues was significantly higher than that in non-cancerous tissues. (B) Curve with color of brown represents the group of low expression of PYCR2, prognosis was also markedly different between patients with CRC with high and low expression levels of PYCR2. Overall survival rate of patients with high expression PYCR2 level was worse. 


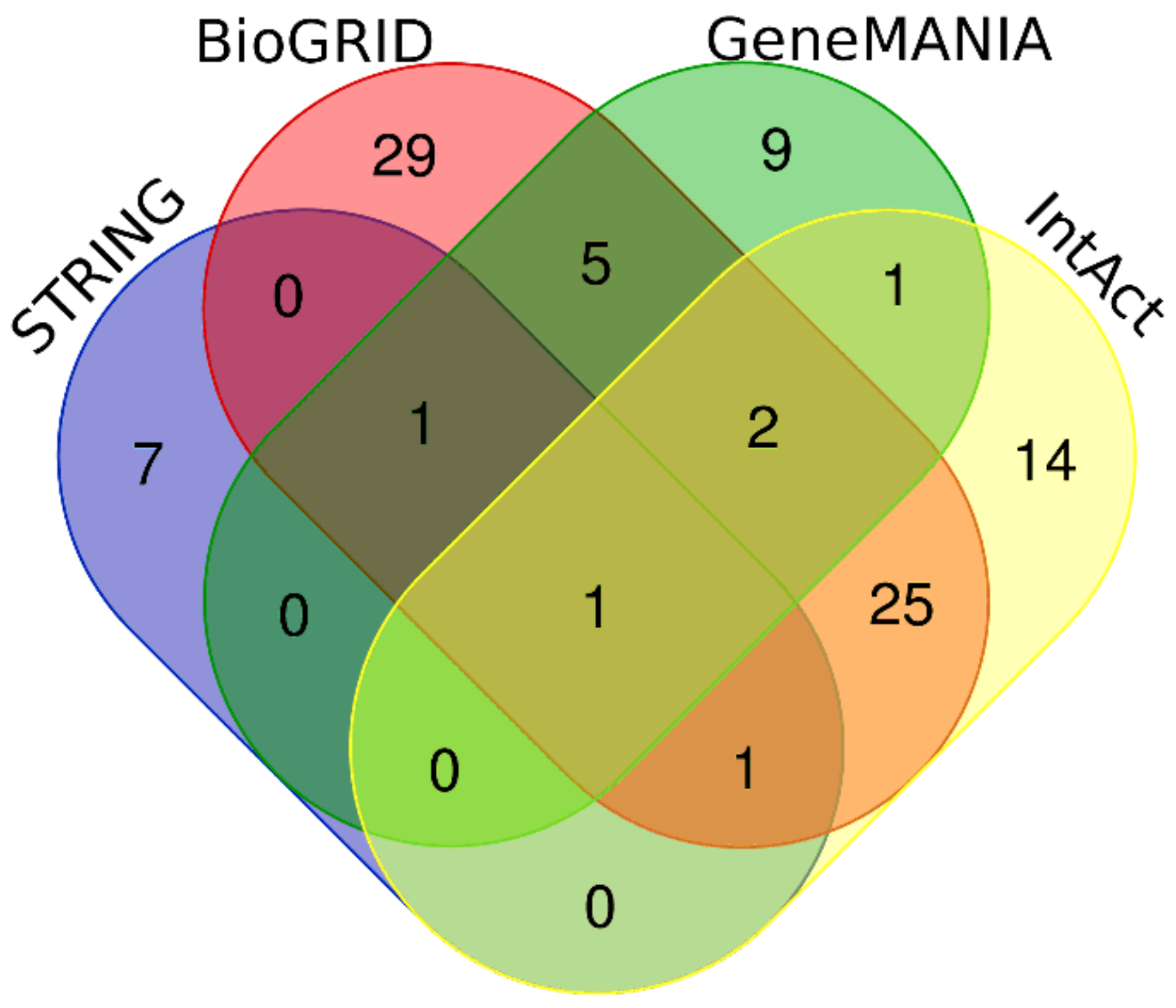

Figure 2

Venn diagram of target genes predicted from STRING, BioGRID, IntAct, and GeneMANIA. Different colors represent different datasets. 
A

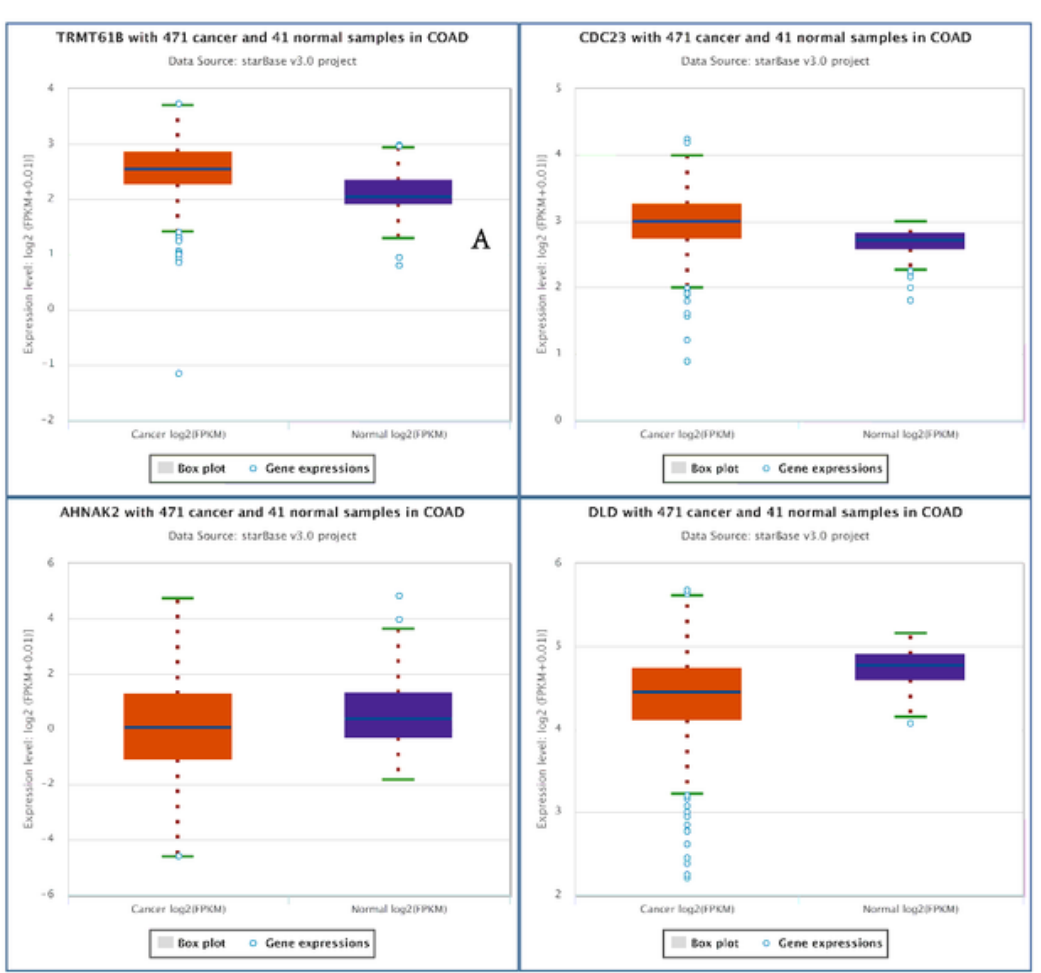

B
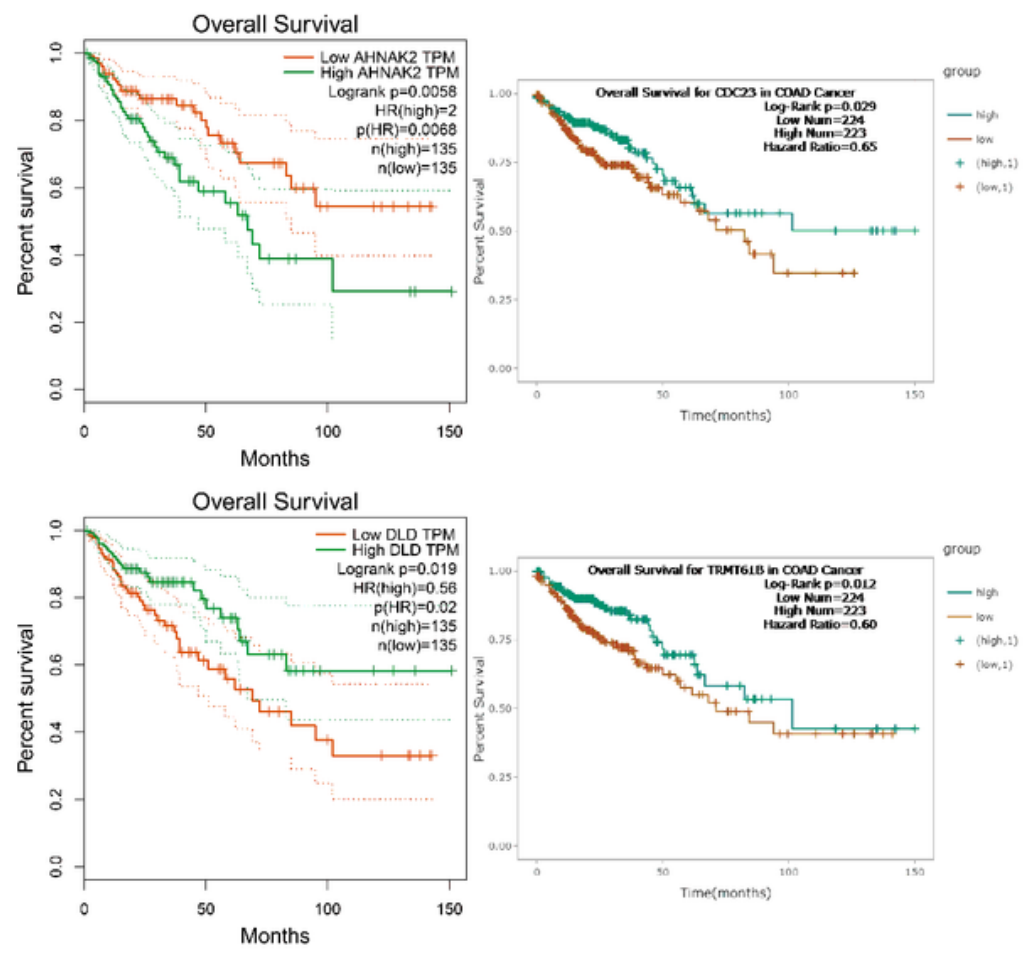

\section{Figure 3}

Expression levels and prognosis-related information of selected genes in CRC and non-cancerous tissues. (A) Four of the 36 identified genes are significantly altered both in terms of their expression and prognostic status $(\mathrm{p}<0.05)$. Orange and purple represent $C R C$ and non-cancerous tissues, respectively. (B) Online Kaplan-Meier plotting tools were used to gather prognostic information. TRMT61B, CDC23, AHNAK2, and DLD were closely associated with survival rates $(p<0.05)$. 


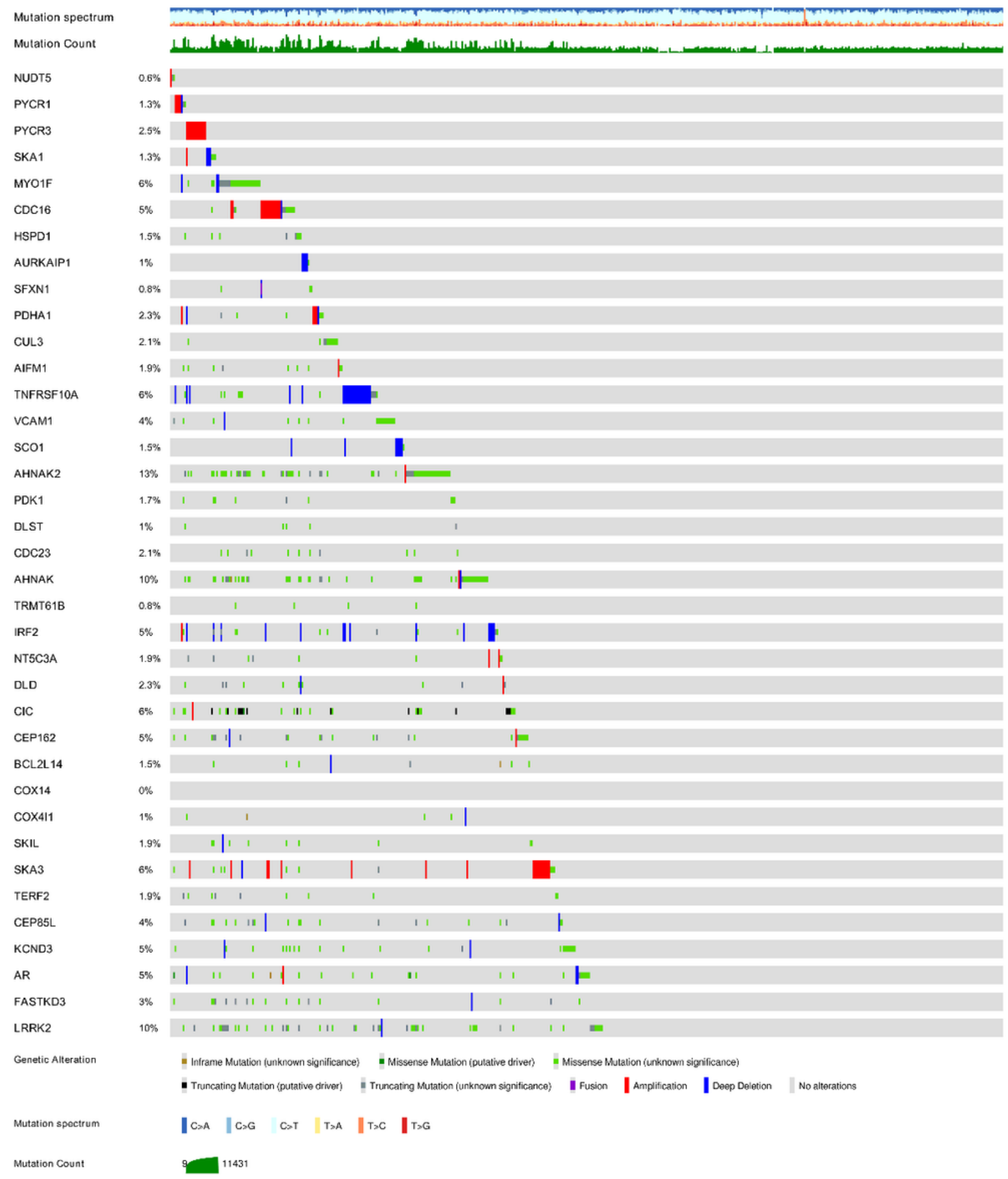

Figure 4

Mutation frequency of genes. The mutation status of the 36 identified genes is shown. The genes such as AHNAK2, AHNAK, LRRK2, MYO1F, and CIC showed a high mutation frequency. 


\section{Overall Survival}

Log-Rank Test P-Value $=4.68 \mathrm{e}-2$

$S_{1}(\mathrm{~N}=305)$ - MYO1F Not Mutated Donors Colorectal, Colorectal,

Uploaded gene set

$S_{2}(\mathrm{~N}-\mathrm{a}(\mathrm{f})$ - MYO1F Mutated Donors Colorectal, Colorectal,

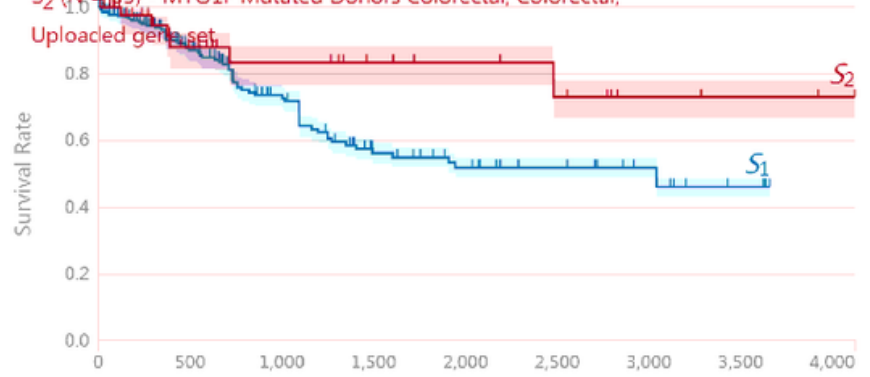

\section{Overall Survival}

Log-Rank Test P-Value $=8.29 \mathrm{e}-2$

$S_{1}(\mathrm{~N}=23)$ - IRF2 Mutated Donors Colorectal, Colorectal, Uploaded

gene set

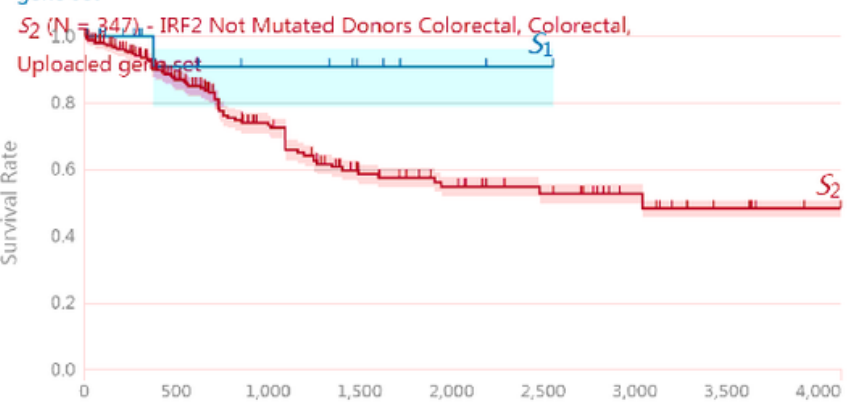

\section{Overall Survival}

Log-Rank Test P-Value $=7.50 \mathrm{e}-2$

$S_{1}(\mathrm{~N}=33)$ - DLD Mutated Donors Colorectal, Colorectal, Uploaded

gene set

$\left.S_{2}(1)=3.37\right)$ - DLD Not Mutated Donors Colorectal, Colorectal,

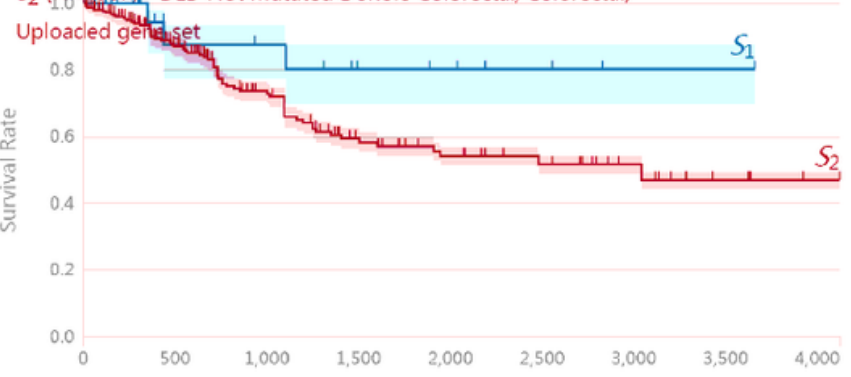

Duration (days)

\section{Overall Survival}

Log-Rank Test P-Value $=4.21 \mathrm{e}-2$

$S_{1}(\mathrm{~N}=310)$ - CIC Not Mutated Donors Colorectal, 1 Colorectal,

Uploaded gene set
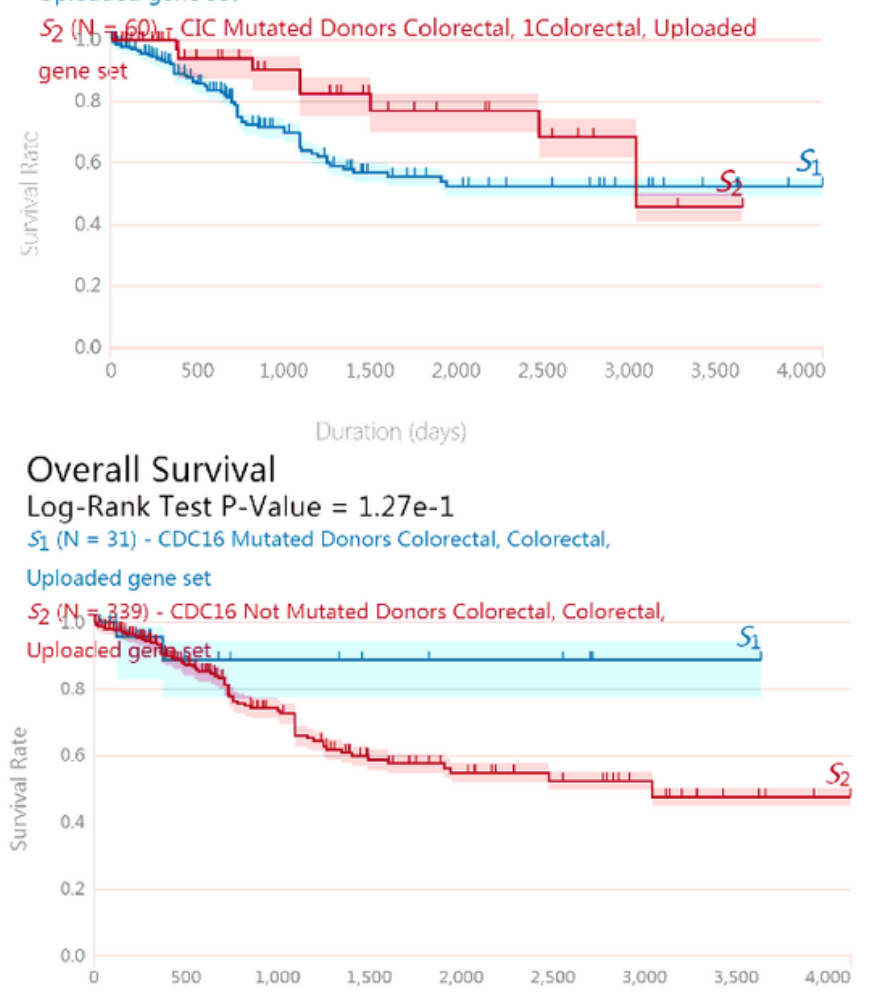

\section{Overall Survival}

Duration (days)

Log-Rank Test P-Value $=9.98 \mathrm{e}-2$

$S_{1}(\mathrm{~N}=332)$ - FASTKD3 Not Mutated Donors Colorectal, Colorectal,

Uploaded gene set

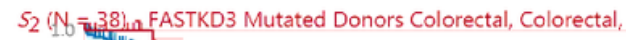

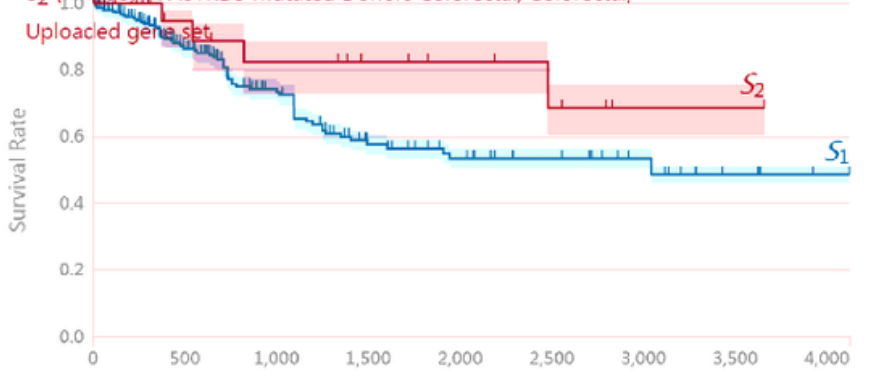

Duration (days)

\section{Figure 5}

Prognosis between patients with and without gene mutations. CIC and MY01F showed a significant impact on the prognosis of CRC $(P<0.05)$. The overall survival curves of CDC16, DLD, IRF2, and FASTKD showed a significant change, but $P$ was not statistically significant. 
A
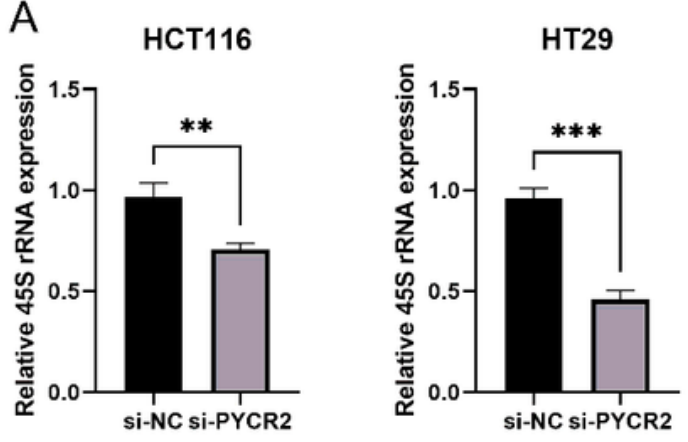

B

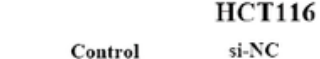

HCT11
si-NC

si-PYCR2
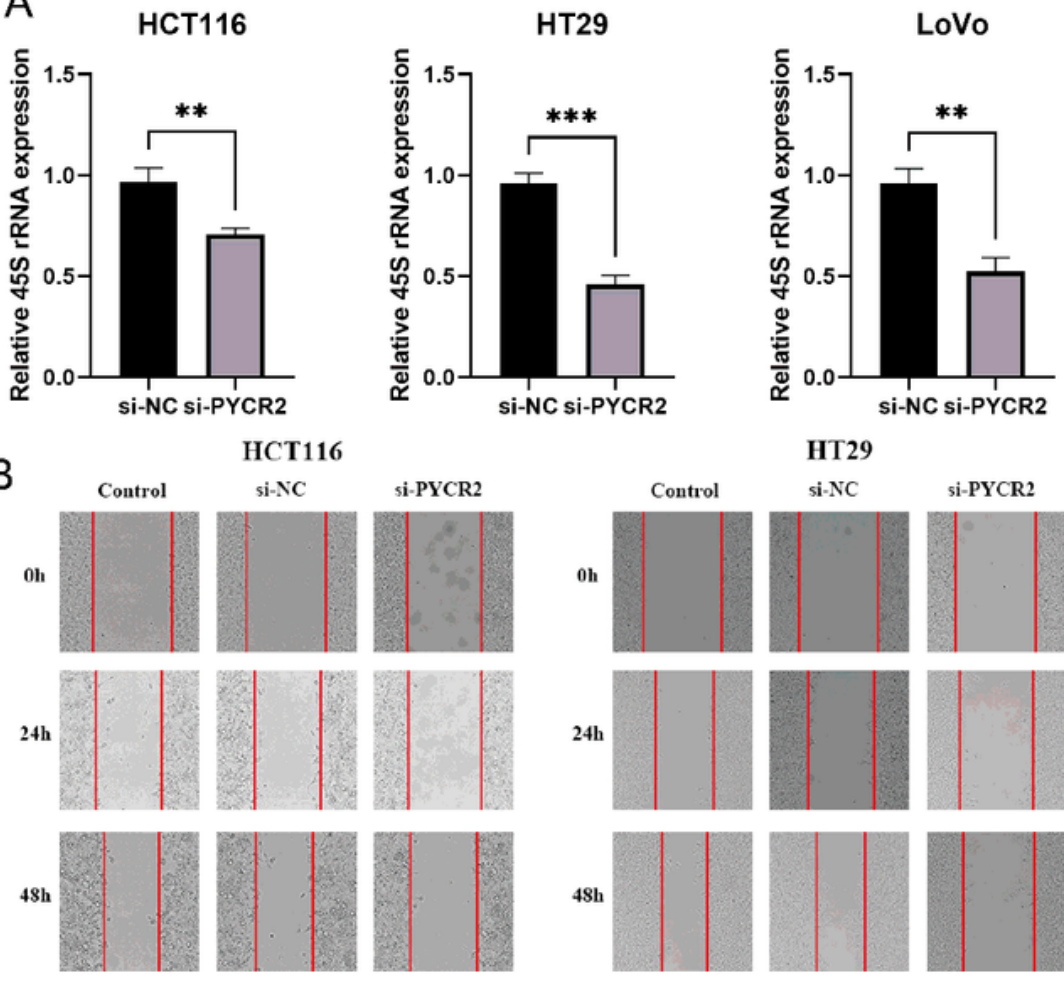

HT29

si-NC

si-PYCR2
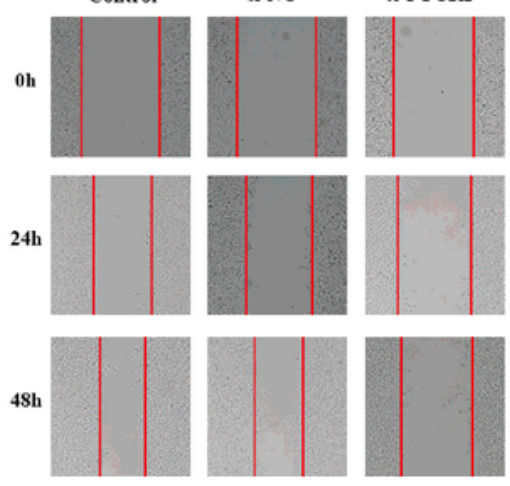

\section{LoVo}
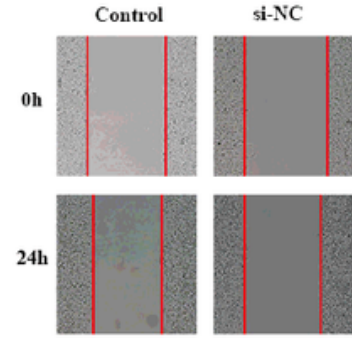

si-PYCR2

48h
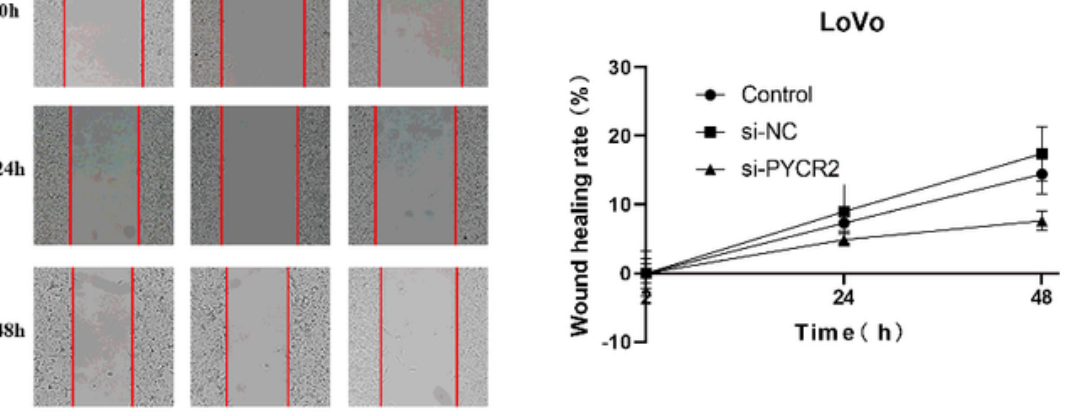

C

HCT116
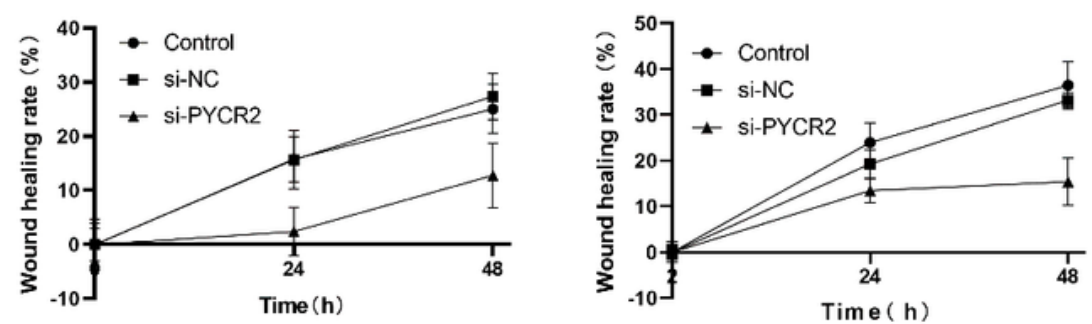

Figure 6

Effect of PYCR2 on CRC cell migration. (A) PYCR2 expression levels were detected using reverse transcription-quantitative PCR following transfection of HCT116, HT29, and LoVo cells with si NC and si PYCR2. (B) Migration ability of cells was examined using wounding healing assay. (C) 48-h healing ratio. (D) Representative images of the Transwell assay (magnification, $x 200$ ). (E) Number of migrated cells after 48 h. NC, negative control. ** $p<0.01 ;{ }^{* \star *} p<0.001$. 